\title{
Complex cardiovascular defects in a male infant with Williams syndrome juxtaposed with the results of a preliminary survey illustrating other patients' outcomes
}

\author{
1 Department of Pediatrics, Oncology and Hematology, Medical University of Lodz, tódź, Poland \\ 2 Student's Scientific Association “Prenatal Cardiology," Medical University of Lodz, Łódź, Poland \\ 3 Department of Genetics, Polish Mother's Memorial Hospital Research Institute, Łódź, Poland \\ 4 Department of Cardiology, Polish Mother's Memorial Hospital Research Institute, Łódź, Poland \\ 5 Department of Cardiac Surgery, Polish Mother's Memorial Hospital Research Institute, tódź, Poland \\ 6 Department of Diagnosis and Prevention Fetal Malformations, Medical University of Lodz, Łódź, Poland \\ 7 Department of Prenatal Cardiology, Polish Mother's Memorial Hospital Research Institute, tódź, Poland
}

Bartosz Szmyd ${ }^{1}$, Filip F. Karuga², Agnieszka Gach³, Tomasz Moszura4 ${ }^{4}$ Marek Kopala5, Maria Respondek-Liberska ${ }^{6,7}$

Correspondence to: Bartosz Szmyd, Department of Pediatrics, Oncology and Hematology, Medical University of Lodz, ul. Sporna 36/50, 91-738 Łódź, Poland, phone: +48426177750 , email: bartoszmyd@gmail.com Received: October 14, 2020. Revision accepted: December 29, 2020 .

Published online: January 7, 2021. Kardiol Pol. 2021; 79 (2): 188-191 doi:10.33963/KP.15740

Copyright by the Author(s), 2021
Introduction Williams syndrome (WS) is a multisystemic disorder that affects connective tissue and both cardiovascular and central nervous systems and is observed in 1/10 000 newborns. It is caused by a hemizygous deletion of a $1.5-1.8 \mathrm{Mb}$ fragment spanning approximately 27 genes on chromosome 7q11.23. ${ }^{1} \mathrm{~Pa}-$ tients with WS have a specific elfin-like phenotype, an ebullient personality profile accompanied by a lower intelligence quotient, and cardiovascular abnormalities. ${ }^{1}$ The latter feature is the direct result of the ELN gene deletion. The gene product, called elastin, is a crucial building component of connective tissue and large, elastic blood vessels. It accounts for approximately a half of the total dry weight of the aorta. ${ }^{2}$ In arteries, elastin is responsible for the appropriate propagation of the pressure wave. The ELN hemizygous deletion leads to higher arterial stiffness associated with cardiovascular abnormalities. Thus, cardiovascular manifestations, especially arterial stenosis: supravalvular aortic stenosis $(45 \%-75 \%)$ and pulmonary artery stenosis (37\%-75\%), are observed in almost $80 \%$ of patients with WS. ${ }^{1}$ Here, we present a case study of complex cardiovascular defects in a male infant with WS, juxtaposed with survey results illustrating other patients' outcomes.
Methods We analyzed the case of a male infant with complex cardiovascular defects diagnosed prenatally, in whom a postnatal genetic diagnosis of WS was established. Our patient required long and complex treatment by interventional cardiologists and cardiac surgeons. To assess the prevalence and severity of cardiac disease in Polish patients with WS, we prepared a questionnaire using Google Forms. It comprised questions concerning the following data: age and sex of the patient with WS, mother's and father's age at delivery, a familial history of WS, the patient's age at the first cardiac consultation, detected cardiovascular abnormalities and treatment options used, the patient's age at the time of WS diagnosis, and abnormalities observed before the diagnosis. The survey was distributed among the members of the Polish Williams Syndrome Association via the Facebook fan page. The study was approved by the ethics committee. All patients and/or patients' parents provided written informed consent to participate in the study.

Statistical analysis The normality of data distribution was assessed with the Shapiro-Wilk test, using the Statistica software (StatSoft, Tulsa, Oklahoma, United States). As all numerical variables did not have normal distrubution, 


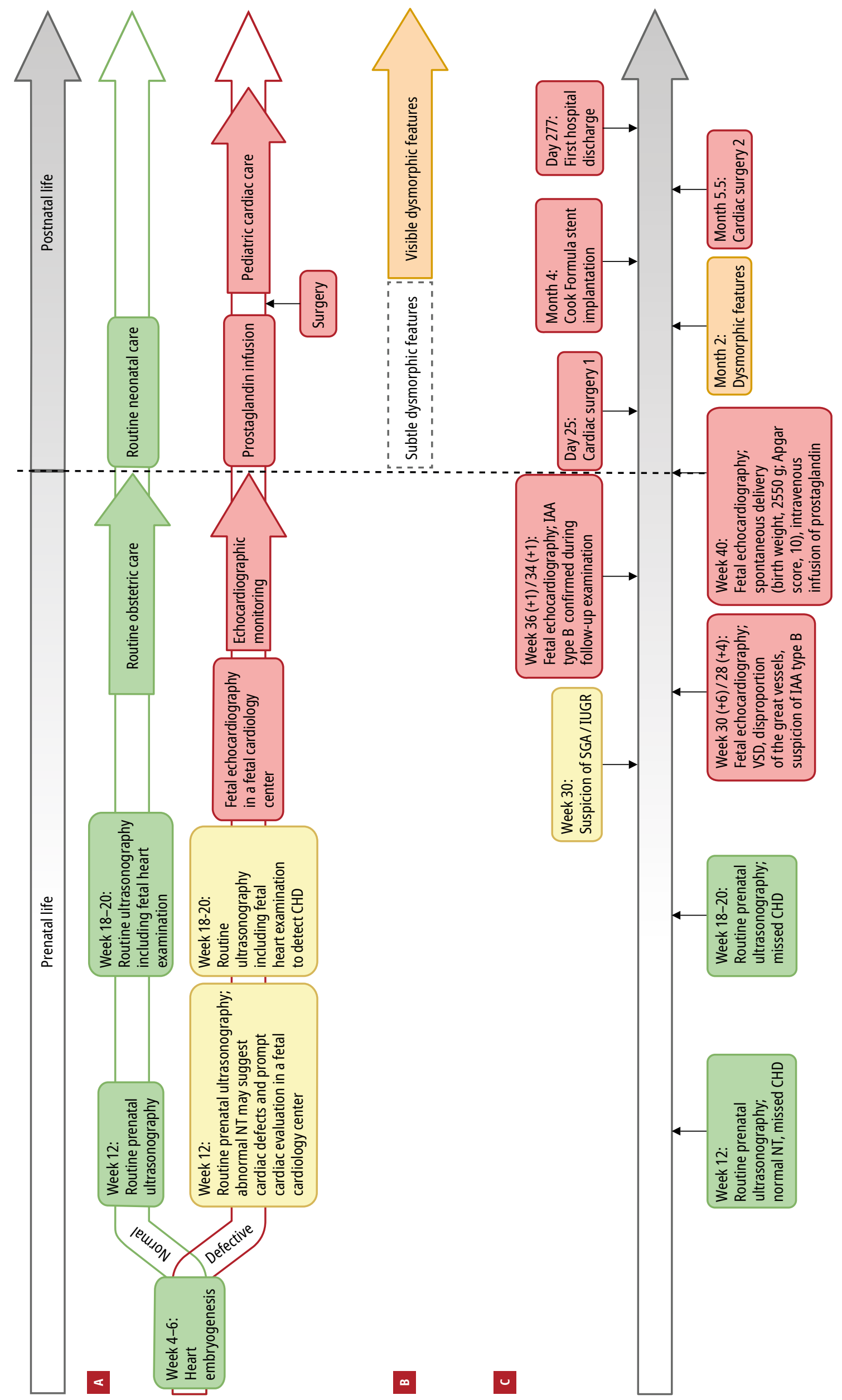

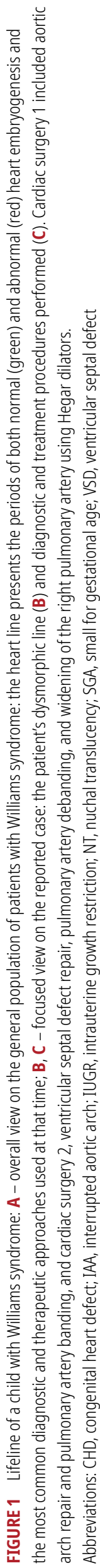


data were presented as median and interquartile range (IQR). Qualitative variables were expressed as number and percentage.

Results and discussion Case study A 34-year-old gravida 3, para 3, in the third trimester, was referred to the Department of Prenatal Cardiology, as the fetus was expected to be small for gestational age (SGA) and had intrauterine growth restriction (IUGR) detected by ultrasound at 30 weeks of gestation (wg). The 4-chamber view of the heart suggested further abnormalities. The fetus appeared normal on previous ultrasound examinations performed by a primary care obstetrician at 12 and $20 \mathrm{wg}$, except for an abnormal heart (FIGURE 1A). Fetal echocardiography performed at our department at $30 \mathrm{wg}$ revealed a complex heart defect including symmetrical atria and ventricles and a ventricular septal defect (VSD) of 4 to $5 \mathrm{~mm}$ in the inlet region. ${ }^{3}$ Moreover, large vessel disproportion was observed. The wide pulmonary trunk $(8 \mathrm{~mm})$ passed into the ductus arteriosus of hockey-stick shape. The narrow aorta with normal transaortic flow was directed cephalically, which was identified as type B interrupted aortic arch (IAA). A follow-up examination performed in our department at $36 \mathrm{wg}$ confirmed the presence of the defects (FIGURE 1C). The following recommendations were made based on prenatal findings: 1) planned delivery in a reference center; 2) prostaglandin administration in the neonate; and 3) planned cardiac diagnostic workup before cardiac surgery.

At week 40, the mother spontaneously delivered a boy weighing 2550 g, with an Apgar score of 10. Intravenous infusion of prostaglandin was immediately administered via the umbilical vein. No dysmorphic features were noted in the newborn at that time. The neonate underwent aortic arch repair and pulmonary artery banding on day 25. Aortic reconstruction was performed using only the patient's own tissue to promote the growth and prevent stenotic lesions. The ductus arteriosus was completely excised, and the remnants of the ductal tissue in the descending aorta were removed. The ascending aorta and the proximal part of the arch up to the left carotid artery were longitudinally incised. End-to-side anastomosis was performed using the Prolene 7.0 suture. The postoperative period was complicated by several events: edema, oliguria, supraventricular tachycardia, and pneumonia. We also observed recurrent hypokalemia and anemia. Subtle dysmorphic features were observed, and a clinical geneticist suggested the diagnosis of WS, which was later confirmed (FIGURE 1B and 1 C).

At the age of 4.5 months, due to 2-mm isthmic aortic stenosis, the Cook Formula stent was placed into the aortic isthmus. The starting gradient in the aortic arch was approximately $40 \mathrm{~mm} \mathrm{Hg}$ with a maximal peak-to-peak gradient in the aortic isthmus of $26 \mathrm{~mm} \mathrm{Hg}$. After surgery, the gradients were as follows: $10 \mathrm{~mm} \mathrm{Hg}$ in the Cook Formula stent and approximately $23 \mathrm{~mm} \mathrm{Hg}$ in the aortic arch (Supplementary material). At the age of 5.5 months, the infant underwent surgical repair of VSD, pulmonary artery debanding, and right pulmonary artery dilation. Finally, the infant was transferred to home hospice care at the age of 277 days.

At the time of writing, the 2-year-old boy was in severe general condition and had a reduced left ventricular ejection fraction of $25 \%$. The gradient in the aortic arch was approximately $90 \mathrm{~mm} \mathrm{Hg}$. The child was deemed eligible for aortic arch reconstruction in Stanford, California, United States, with an estimated risk of death during the procedure of about $50 \%$.

Results of the survey We obtained results from 18 families of patients with WS (including 9 men; Supplementary material, Table S1). The median (IQR) age of patients with WS was 4.6 (2.46-7.5) years. The median (IQR) parents' age at the time of child's birth was 28 (27-31.5) years in mothers and 31.5 (29-35.75) years in fathers. Williams syndrome was diagnosed only postnatally at the median (IQR) age of 13 (8.25-17.75) months. The main reasons for further genetic testing leading to the diagnosis of WS were dysmorphia (77.78\%), cardiovascular abnormalities $(72.22 \%)$, slow or poor infant weight gain and / or short stature (33.33\%), psychomotor development retardation (27.78\%), and hypothyroidism (16.67\%). Other reported signs included constipation, enamel defects, hernias, hydrocele, a protruding mandible, and renal abnormalities. No family had a positive history of WS.

All patients underwent cardiac examination. The first cardiac consultation was performed at the child's median (IQR) age of $1(0-3)$ month, preceding the final diagnosis by years. The most prevalent cardiovascular abnormalities were pulmonary artery stenosis (61.11\%), aortic stenosis (38.89\%), VSD (11.11\%), and aortic valve defects (11.11\%). Other abnormalities included cyanosis, hypertrophic cardiomyopathy, mitral valve defect, murmurs, patent ductus arteriosus, pulmonary valve defect, and aneurysm. The characteristics of patients requiring interventional cardiological procedures and/or cardiac surgical treatment are presented in Supplementary material, Table S1. ${ }^{4}$

Discussion The first finding indicative of WS in our patient was abnormal fetal biometry suggesting that the infant was small for gestational age and had IUGR observed since $30 \mathrm{wg}$. This is in line with Marcato et al, ${ }^{5}$ who presented a series of 3 cases with prenatal phenotypes of WS. In all of those cases, IUGR was detected at different stages of prenatal development $(18,20$, and $32 \mathrm{wg}) .^{5}$ Other researchers have also reported IUGR in fetuses with WS phenotype at 20 and 25 wg. ${ }^{6,7} \mathrm{In}-$ terestingly, residual symptoms in postnatal life 
such as slow or poor infant weight gain and/or short stature were reasons for further genetic testing in $33.33 \%$ of our patients. According to other studies, approximately $70 \%$ of patients with WS had prenatal growth deficiency and did not thrive in infancy. ${ }^{8}$

Complex cardiovascular defects including VSD, great vessel disproportion, and narrow aorta with type B IAA were another prenatal symptoms suggestive of WS. Interestingly, cardiac abnormalities were only observed in 1 out of 3 patients described in a study by Marcato et al, ${ }^{5}$ in which aortic stenosis was detected at $32 \mathrm{wg}$. Only $33 \%$ of diagnosed cardiovascular defects may be detected prenatally and they tend to be mild. For instance, aortic stenosis or pulmonary stenosis might be missed by routine ultrasound examinations. ${ }^{6,7}$ The fact that at least $80 \%$ of patients with WS had cardiovascular abnormalities in their postnatal life highlights the significance of the effective prenatal and postnatal examination of the cardiovascular system. The patients with WS in our study group had their first cardiac examination performed relatively early, in the first month (IQR, 0-3 months) after birth, and cardiovascular abnormalities were one of the key reasons for further genetic testing.

The specific "elfin-like" phenotype was the main indication for postnatal, "late" genetic counseling among patients in our survey. It was reported in $77.78 \%$ of all cases. This is in line with other studies, which indicates that both WS-specific phenotype and cardiovascular abnormalities remain the basis for the diagnosis of WS. ${ }^{1}$

Most frequently reported cardiovascular abnormalities include pulmonary artery stenosis (61.11\%), aortic stenosis (38.89\%), VSD (11.11\%), and aortic valve defects (11.11\%). Our data correspond with findings from other studies, which show that the majority of patients with WS present with arterial stenosis, namely supravalvular aortic stenosis $(45 \%-75 \%)$ and pulmonary artery stenosis (37\%-75\%). ${ }^{1}$

Conclusions Our study showed that being small for gestational age and / or having IUGR accompanied by cardiovascular defects were the main WS abnormalities detected prenatally. Cardiovascular abnormalities in WS have a broader spectrum than described in the literature so far.

\section{SUPPLEMENTARY MATERIAL}

Supplementary material is available at www.mp.pl/kardiologiapolska.

\section{ARTICLE INFORMATION}

ACKNOWLEDGMENTS We would like to thank the patients and their legal representatives for participation. Moreover, we express our gratitude to Aleksandra Sobieska-Listewnik from the Polish Williams Syndrome Association for distribution of questionnaires among the members of their association.

CONTRIBUTION STATEMENT MR-L, AG, TM, and MK performed the clinical examination. BSz and FK collected clinical data. BSz wrote the manuscript. MR-L, FFK, AG, TM, and MK revised the manuscript. All authors accepted the final version of the manuscript.

CONFLICT OF INTEREST None declared.
OPEN ACCESS This is an Open Access article distributed under the terms of the Creative Commons Attribution-NonCommercial-NoDerivatives 4.0 International License (CC BY-NC-ND 4.0), allowing third parties to download articles and share them with others, provided the original work is properly cited, not changed in any way, distributed under the same license, and used for noncommercial purposes only. For commercial use, please contact the journal office at kardiologiapolska@ptkardio.pl.

HOW TO CITE Szmyd B, Karuga F, Gach A, et al. Complex cardiovascular defects in a male infant with Williams syndrome juxtaposed with the results of a preliminary survey illustrating other patients' outcomes. Kardiol Pol. 2021; 79: 188-191. doi:10.33963/KP.15740

\section{REFERENCES}

1 Collins RT. Cardiovascular disease in Williams syndrome. Circulation. 2013; 127: 2125-2134.

2 Leung DYM, Glagov S, Mathews MB. Elastin and collagen accumulation in rabbit ascending aorta and pulmonary trunk during postnatal growth. Correlation of cellular synthetic response with medial tension. Circ Res. 1977; 41: 316-323.

3 Bokiniec R, Własienko P, Szymkiewicz-Dangel J, Borszewska-Kornacka MK. Echocardiographic analysis of left ventricular function in term and preterm neonates at week 40 of postconceptional life. Kardiol Pol. 2019; 77: 445-450.

4 Płońska-Gościniak E, Suwalski P, Bartuś S, et al. Valvular and structural heart disease management during the COVID-19 pandemic. Expert opinion of the Working Group on Valvular Heart Diseases, the Working Group on Cardiac Surgery, and the Association of Cardiovascular Interventions of the Polish Cardiac Society. Kardiol Pol. 2020; 78: 498-507.

5 Marcato L, Turolla L, Pompilii E, et al. Prenatal phenotype of Williams-Beuren syndrome and of the reciprocal duplication syndrome. Clin Case Reports. 2014; 2: 25-32.

6 Krzeminska D, Steinfeld C, Cloez JL, et al. Prenatal diagnosis of Williams syndrome based on ultrasound signs. Prenat Diagn. 2009; 29: 710-712.

7 Popowski T, Vialard F, Leroy B, et al. Williams-Beuren syndrome: the prenatal phenotype. Am J Obstet Gynecol. 2011; 205: e6.

8 Morris CA. Williams Syndrome. GeneReviews ${ }^{\circledR}$. http://www.ncbi.nlm.nih. gov/pubmed/20301427. Accessed October 8, 2020. 